www.nature.com/pj

\title{
A two-dimensional spin-diffusion NMR study on the local structure of a water-soluble model peptide for Nephila clavipes dragline silk (MaSp1) before and after spinning
}

\author{
Koji Yazawa, Erika Yamaguchi, Akihiro Aoki, Yasumoto Nakazawa, Yuu Suzuki and Tetsuo Asakura
}

Polymer Journal (2012) 44, 913-917; doi:10.1038/pj.2012.98; published online 13 June 2012

Keywords: ${ }^{13} \mathrm{C}$ proton-driven 2D spin-diffusion; model peptide; NMR; Spider silk

\section{INTRODUCTION}

The dragline silk of the golden orb web spider Nephila clavipes has received significant attention because of its remarkable mechanical properties, which include toughness and high tensile strength. ${ }^{1,2}$ This silk contains two structural proteins designated as major ampullate spidroin 1 (MaSp1) and major ampullate spidroin 2 (MaSp2). ${ }^{3,4}$ The dominant MaSp1 can be described as $\mathrm{AB}$ block-copolymers consisting of numerous alternating poly-Ala (A) and Gly-rich (B) blocks. The dragline silk fiber is produced from an aqueous spinning solution (dope) containing $\sim 20 \% \mathrm{w} / \mathrm{v}$ spidroins at ambient pressure and temperature with substantial conformational change. Several factors have been thought to be important for controlling this conformational change, including mechanical strain, the concentration of fibroin, changes in $\mathrm{pH}$, and the concentration of other ions. ${ }^{5,6} \mathrm{~A} \mathrm{pH}$ change from $6.9 \pm 0.1$ to $6.3 \pm 0.1$ from the start of the silk gland duct to the spigot is crucial for gelling the fibroin, increasing its sensitivity to shear, and initiating the conformation transition. . $6,7^{2}$

We previously performed solid-state NMR structural analysis of ${ }^{13} \mathrm{C}$ selectively labeled versions of the water-soluble model peptide, (E) ${ }_{8}$ GGLGGQGAG(A) ${ }_{6}$ GGAGQGG-YGG, to gain insight about the local structure of the MaSpl protein before and after spinning. ${ }^{8}$ Polyglutamic acid $(\mathrm{E})_{8}$ included at the amino terminus makes the peptide water soluble, leading to mimicking the predominance of amino acids with acidic side chains in this part of the sequence in spider fibroins and the induction of the conformational transition by lowering the $\mathrm{pH} .{ }^{6,9}$ One-dimensional ${ }^{13} \mathrm{C}$-cross polarization magic angle-spinning NMR spectra of the lyophilized and acid-treated peptide samples revealed that the conformational change induced by acidification primarily occurred in the poly-Ala, and to a lesser extent in the GAGA and GGA sequences. Moreover, the amount of $\beta$-sheet was the largest in the center of the poly-Ala domain compared with its periphery. These observations suggest that the peptide can be used as a model for studying the effects of acidification on changes in the local conformation. ${ }^{8}$ However, further detailed structures, such as the torsion angles in the $\beta$-sheet fraction, are still unclear.

Two-dimensional (2D) proton-driven spin-diffusion solid-state NMR under off-magic-angle spinning (OMAS) coupled with ${ }^{13} \mathrm{C}$ isotope double labeling of specific residues has been used as a powerful method for determining the torsion angles of the backbone amino-acid residues for the silk model peptides. ${ }^{10-18}$ The combination with rotational-echo double-resonance measurements is a more quantitative approach, as previously reported, ${ }^{10,15,18}$ but it is not easy to quantitatively determine the structure when the structure is heterogeneous, such as a mixture of $\beta$-sheets and random coil conformations. In this case, it is better to first rigorously determine the fraction of the mixed conformations of individual residues. We should then try to determine the structure using spin-diffusion solidstate NMR by taking the fraction into account.

In this paper, we will propose an analytical method for estimating the detailed local structures in the conformational ensemble system using 2D spin-diffusion OMAS NMR experiments combined with information about the fractions of different conformations at specific sites obtained from the 1D spectra. ${ }^{8}$ Furthermore, we will also discuss the detailed structures of the acid-treated model peptide, such as the torsion angles in the $\beta$-sheet component.

\section{EXPERIMENTAL PROCEDURE}

Three types of ${ }^{13} \mathrm{C}$ selectively double-labeled carbonyl carbon sites of the water-soluble spider silk model peptides with the same structure were designed to obtain detailed information on the local conformation of the Ala residue, as summarized in Table 1. The labeled residues in the P1, P2 and P3 are located at $\mathrm{Gly}^{17}-\mathrm{Ala}^{18}, \mathrm{Ala}^{20}-\mathrm{Ala}^{21}$, and $\mathrm{Gly}^{25}-\mathrm{Ala}^{26}$, respectively, and consequently, the torsion angles of the $\mathrm{Ala}^{18}, \mathrm{Ala}^{21}$ and $\mathrm{Ala}^{26}$ residues can be determined using $2 \mathrm{D}$ spin diffusion analysis. These peptides were synthesized using the F-moc 
Table 1 Synthesized ${ }^{13} \mathrm{C}$ double-labeled peptides used for the 2D spin-diffusion analysis

\begin{tabular}{|c|c|c|}
\hline & Peptide & information \\
\hline P1 & $(\mathrm{E})_{8} \mathrm{GGLGGQGA}\left[1-{ }^{13} \mathrm{C}\right] \mathrm{G}\left[1-{ }^{13} \mathrm{C}\right] \mathrm{A}$ AAAAAGGAGQGGYGG & $\operatorname{Ala}^{18}(\varphi, \psi)$ \\
\hline P2 & $(\mathrm{E})_{8} \mathrm{GGLGGQGAGAA}\left[1-{ }^{13} \mathrm{C}\right] \mathrm{A}\left[1-{ }^{13} \mathrm{C}\right] \mathrm{AAAGGAGQGGYGG}$ & $\operatorname{Ala}^{21}(\varphi, \psi)$ \\
\hline P3 & 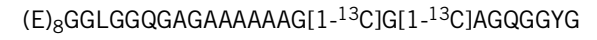 & $\operatorname{Ala}^{26}(\varphi, \psi)$ \\
\hline
\end{tabular}

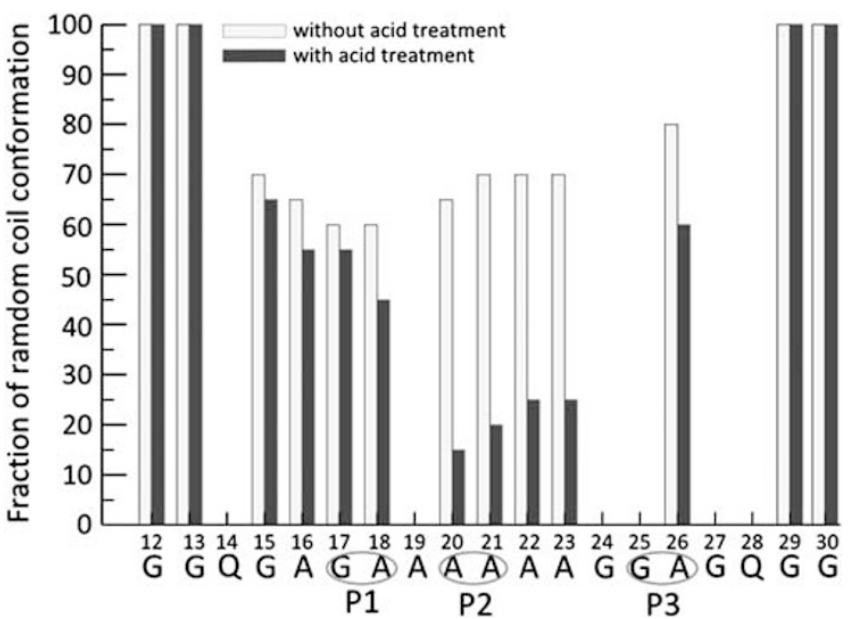

Figure 1 The histogram of the fraction of random coil conformations of each residue in the model peptide for the MaSpl silk protein of the $N$. clavipes dragline silk with poly(glutamic acid) at the amino terminus, (E) $\square$ GGLGGQGAGAAAAAAGGAGQGGYGG, at 'without' (gray) and 'with acid treatment' (black) states, as determined from our previous solid-state NMR studies. ${ }^{8}$ The double ${ }^{13} \mathrm{C}$-labeling sites are marked by circles. The notations, $\mathrm{P} 1-\mathrm{P} 3$, refer to different ${ }^{13} \mathrm{C}$ selectively labeled versions for the peptide. A full color version of this figure is available at Polymer Journal online. solid phase method using a PE Pioneer Peptide Synthesizer (Applied Biosystems, Tokyo, Japan), as described previously. ${ }^{18,19}$ The obtained peptides were purified by reverse-phase high-performance liquid chromatography using a $0.1 \% \mathrm{TFA} / \mathrm{H}_{2} \mathrm{O}$ and $0.08 \%$ TFA/acetonitrile gradient with a PEGASIL ODSII, C-18 column. Two states of the peptides, 'without acid treatment' and 'with acid treatment', were prepared as described previously. ${ }^{8}$ The solid-state NMR experiments were performed on Varian Unity INOVA 400 NMR spectrometer equipped with a $7 \mathrm{~mm}$ Jakobsen-type double-tuned MAS probe. The chemical shifts were indirectly calibrated using the adamantane methylene peak observed at 28.8 p.p.m. relative to the TMS peak. For the 2D spin-diffusion NMR experiments, the OMAS condition was $\theta_{\mathrm{m}}-6.7^{\circ}$, where $\theta_{\mathrm{m}}$ is the magic angle, with sample spinning at $6 \mathrm{kHz}$. The scaling factor of the $2 \mathrm{D}$ spin-diffusion spectra is then $(1 / 2)\left(3 \cos ^{2}\left(\theta_{\mathrm{m}}-6.7^{\circ}\right)-1\right)=0.172$. For cross polarization in the $2 \mathrm{D}$ experiments, a $7.0-\mu \mathrm{s}^{1} \mathrm{H} \pi / 2$ pulse followed by a 1.5 -ms contact time with a 5 -s recycle delay was used. The other NMR experimental conditions and the method for the spectral simulation are as described previously. ${ }^{10}$ The data were processed by applying $100 \mathrm{~Hz}$ Gaussian line broadening to both time domains and zero-filling with a final size of $512 \times 512$ data points. We simulated the $2 \mathrm{D}$ spin-diffusion NMR spectra using a program developed in our laboratory. For simulation of the $2 \mathrm{D}$ spin-diffusion spectra, the calculations were performed using a grid of $10^{\circ}$ for the torsion angles, $\varphi$ and $\psi$ values. To quantitatively demonstrate the difference between the observed and calculated spectra, the root-mean-square deviation (RMSD) for each $(\varphi, \psi)$ pair was defined as

$$
\operatorname{RMSD}(\varphi, \psi)=\sqrt{\sum_{i=1}^{N}\left[E_{i}-\lambda(\varphi, \psi) S_{i}(\phi, \psi)\right]^{2} / N}
$$

where $N$ is the number of intensities analyzed, QUOTE are the intensities of the experimental spectra, $S_{i}(\varphi, \psi)$ are the calculated intensities, and $\lambda(\varphi, \psi)$ is a scaling factor for minimizing the $\operatorname{RMSD}$ at $\operatorname{each}(\varphi, \psi)$

\section{RESULTS AND DISCUSSION}

Figure 1 shows a histogram of the fraction of random coil conformations of each carbon in the model peptide, $\mathrm{E}_{8}$ GGLGGQGA
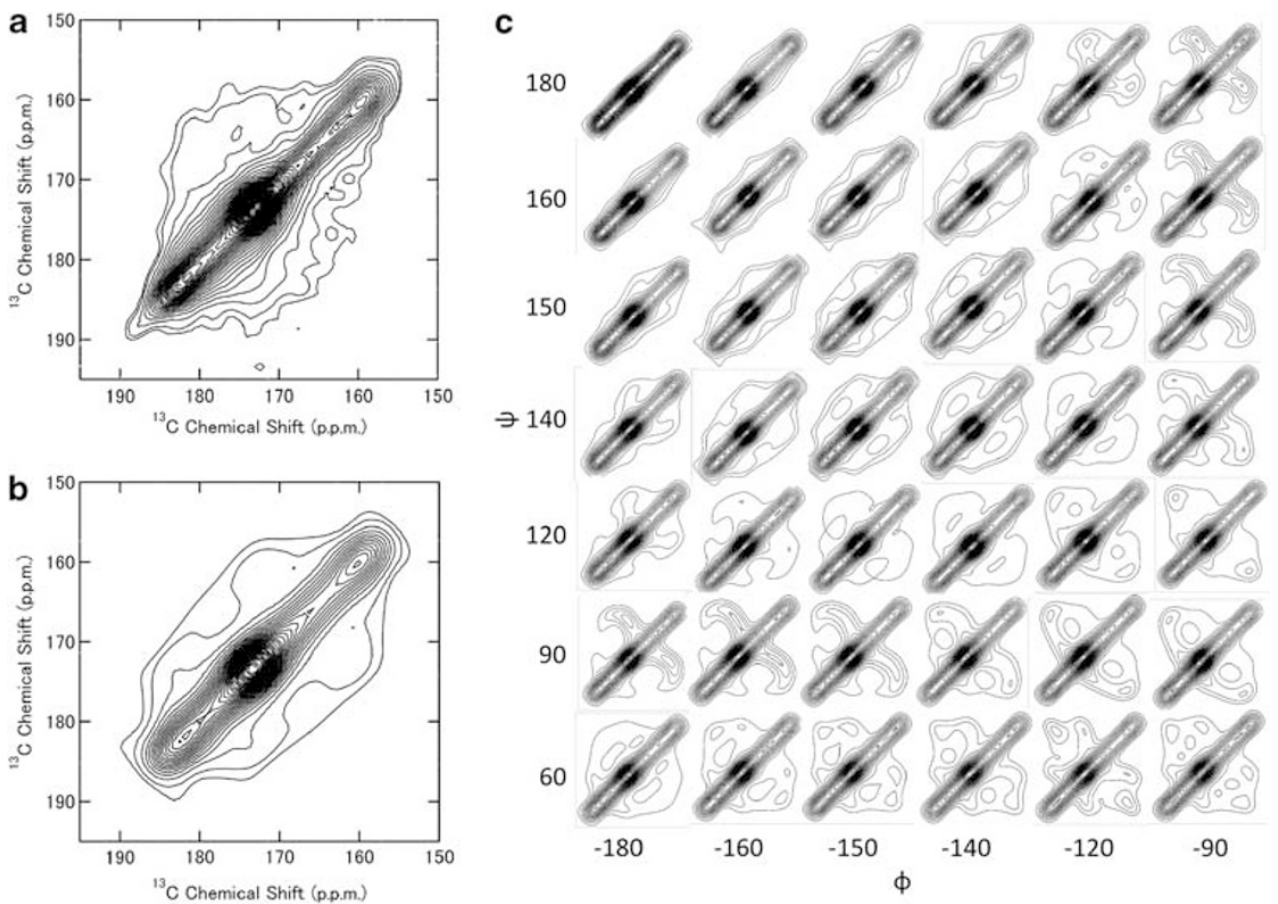

120

Figure 2 (a) Observed and (b) best-fit simulated 2D OMAS spin-diffusion spectra of the model peptide P2 with ${ }^{13} \mathrm{C}$ double-labeled carbonyl carbons of Ala ${ }^{20}$ $\mathrm{Ala}^{21}$. The simulated spectrum is a mixture of the $80 \% \beta$-sheet with a torsion angle of $(\varphi, \psi)=\left(-150^{\circ}, 150^{\circ}\right)$ and $20 \%$ random coil patterns. (c) simulated spectra as a function of the torsion angles, $\varphi$ and $\psi$ of the $\mathrm{Ala}^{21}$ residue in the energetically favored region of $\beta$ sheet, $-180^{\circ}<\varphi<-90^{\circ}, 60^{\circ}<\psi<180^{\circ}$. 
GAAAAAAGGAGQGGYGG, in the 'without acid treatment' (gray) and 'with acid treatment' (black) states, as determined from our previous 1D ${ }^{13} \mathrm{C}{ }^{13} \mathrm{C}$-cross polarization MAS analysis. ${ }^{8}$ Thus, the fraction of random coil conformations of the ${ }^{13} \mathrm{C}$ labeled Ala residue in the model peptides P1-P3 with acid treatment were $\sim 45 \%, 20 \%$ and $60 \%$, respectively. We used these values as a constraint during the simulation of the following ${ }^{13} \mathrm{C}$ proton-driven $2 \mathrm{D}$ spin-diffusion analysis. For the spin-diffusion analysis, we should determine the principal values of the chemical shift tensors of the carbonyl ${ }^{13} \mathrm{C}$ in the Ala and Gly residues, because these values are directly reflected in the simulated peak positions. To adjust the peak positions, we slightly modified the principal values that were reported previously. ${ }^{11}$ The values, in p.p.m., were $\sigma_{11}=247, \sigma_{22}=180, \sigma_{33}=90$ for Ala, and $\sigma_{11}=242, \sigma_{22}=177$, $\sigma_{33}=95$ for Gly. Hereafter, we use these values for the 2D spindiffusion analysis in this paper. Figure $2 \mathrm{a}$ shows the experimental ${ }^{13} \mathrm{C}$ 2D spin-diffusion spectrum of the acid-treated P2, where the carbonyl carbons in the $\mathrm{Ala}^{21}$ and $\mathrm{Ala}^{22}$ residues in the center of the poly-Ala domain were ${ }^{13} \mathrm{C}$ double labeled. To determine the torsion angles in the $\beta$-sheet fraction, we calculated 2D spectra by considering a $20 \%$ random coil fraction. The assumed 2D spectral pattern of the random coil conformation was calculated as a superimposition of the calculated spectral patterns of the Ala residues, which were selected from the energetically allowed region in a Ramachandran map on the basis of stereo-chemical criteria of van der Waals contacts. ${ }^{20}$ Then, the calculated spectrum for comparison with the observed one was calculated by considering an assumed random coil spectral pattern $(20 \%)$ and $\beta$-sheet spectral pattern $(80 \%)$, where the latter was calculated as a function of the torsion angle $(\varphi, \psi)$ of the Ala residue selected from the $\beta$-sheet-favored region $\left(-180^{\circ}<\varphi<-90^{\circ}\right.$, $60^{\circ}<\psi<180^{\circ}$ ). The calculated spectral patterns significantly changed with the torsion angles, $\varphi$ and $\psi$, as shown in Figure 2c. The RMSD between the calculated and observed spectra was shown as a contour map in Figure 3. The best fitted spectrum (Figure $2 \mathrm{~b}$ ) was obtained with a torsion angle of $(\varphi, \psi)=\left(-150^{\circ}, 150^{\circ}\right)$ in the $\beta$ sheet region, as observed in the contour map. The obtained torsion angle is almost the same as the previous one. ${ }^{11}$ Here, the experimental error was $\pm 10^{\circ}$.

We performed a similar approach for the acid-treated P1 and P3 samples. Namely, the ${ }^{13} \mathrm{C}$ double-labeled carbonyl carbons are the $\mathrm{Gly}^{17}-\mathrm{Ala}^{18}$ part at the amino terminus of the poly-Ala region and the $\mathrm{Gly}^{25}-\mathrm{Ala}^{26}$ part in the sequence, GGA adjacent to the carboxyterminal end of the poly-Ala domain, respectively. Figure 4a shows the observed 2D spin-diffusion spectra of $\mathrm{P} 1$ and $\mathrm{P} 3$. The $\mathrm{S} / \mathrm{N}$ ratio of the P1 spectrum was low because of the lack of sample amount, and therefore, the spectral pattern looks slightly asymmetric because of the difficulty with phase correction. The RMSD between the calculated and observed spectra was shown as a contour map in Figure 5. Minimal RMSD values were distributed from the typical torsion angle of the $\beta$-sheet, $(\varphi, \psi)=\left(-150^{\circ}, 150^{\circ}\right)$, to the energetically allowed region other than the $\beta$-sheet-favored region. This result is different from the case of $\mathrm{P} 2$, which is in the center of the poly-Ala region. However, because of the low quality of the spectral pattern for P1, it appears difficult to clearly describe this difference. In the case of P3, where the observed spectral pattern is relatively good, a similar tendency was also observed, indicating a distribution in the energetically allowed region other than the $\beta$-sheet-favored region. Thus, this tendency seems common in both P1 and P3. The best-fitted spectra were obtained from the contour plot of RMSD shown in Figure $4(\mathrm{~b})$. The torsion angles in the $\beta$-sheet-favored region were $(\varphi, \psi)=\left(-130^{\circ}, 150^{\circ}\right)$ for P1 and $(\varphi, \psi)=\left(-180^{\circ}, 140^{\circ}\right)$ for P3. As expected, these values are different from the typical values of a $\beta$-sheet, $(\varphi, \psi)=\left(-150^{\circ}, 150^{\circ}\right)$, which were obtained in the center of

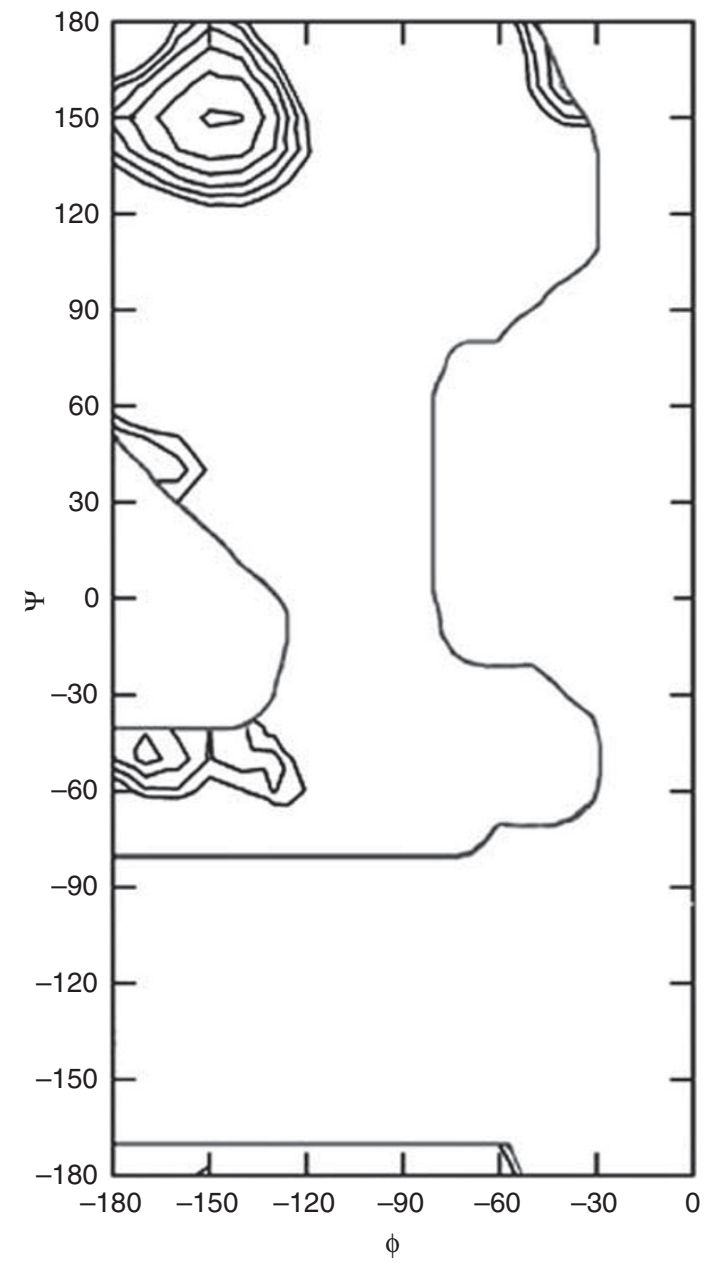

Figure 3 RMSD contour plot for the 2D spin-diffusion of P2 in the energetically allowed region. The torsion angle $(\varphi, \psi)=\left(-150^{\circ}, 150^{\circ}\right)$ gave a good agreement between the observed and calculated 2D spin-diffusion spectra (Figure 2).

the poly-Ala region, as mentioned above. This result also seems to be related with the difference in the random coil fraction, that is, P1 (45\%) and P3 (60\%), compared with P2 (20\%). These results provide insight into the detailed structure of the Ala residues around the $\beta$-sheet poly-Ala region. When apart from the poly-Ala region, distortion of the torsion angle from the typical $\beta$-sheet structure occurred together with an increase in the random coil fraction, as is expected. This paper more quantitatively analyzed the phenomena that occurred locally along the chain of spider silk.

\section{SUMMARY}

The local structure of the acid-treated model peptides, (E) ${ }_{8}$ GGLGGQGAG(A) ${ }_{6}$ GGAGQGGYGG, that were derived from the consensus sequence of the Nephyla edulis fibroin MaSpl was determined using $2 \mathrm{D}$ proton-driven spin-diffusion solid-state NMR under OMAS coupled with ${ }^{13} \mathrm{C}$ isotope double labeling of specific residues. We estimated the torsion angles of the Ala residue in the center of poly-Ala region and of the Ala residues around the poly-Ala region. The positional dependence in the torsion angles of the Ala residues was obtained. These results, together with previous data of the random coil fraction, could more quantitatively indicate that the phenomena occurred locally along the chain of spider silk. 

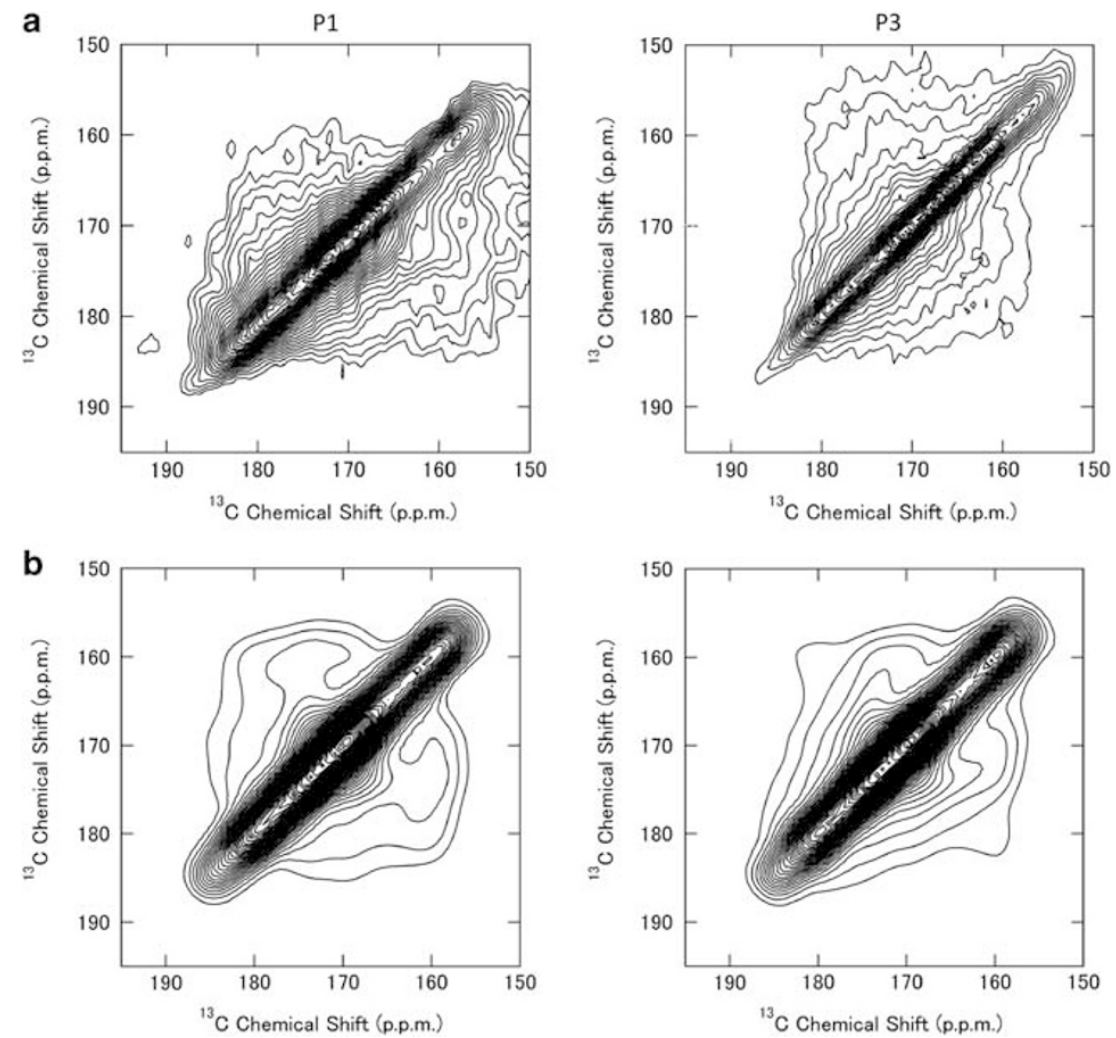

Figure 4 (a) Observed and (b) simulated 2D OMAS spin-diffusion spectra of the model peptide P1 and P3 with ${ }^{13} \mathrm{C}$ double-labeled carbonyl carbons of Gly $^{17}-\mathrm{Ala}^{18}$ and Gly ${ }^{25}-\mathrm{Ala}^{26}$, respectively. The simulated spectra are mixtures of the $55 \% \beta$-sheet with a torsion angle of $(\varphi, \psi)=\left(-130^{\circ}, 150^{\circ}\right)$ and $45 \%$ random coil patterns for $\mathrm{P} 1$, and $40 \% \beta$-sheet with a torsion angle of $(\varphi, \psi)=\left(-180^{\circ}, 140^{\circ}\right)$ and $60 \%$ random coil patterns.
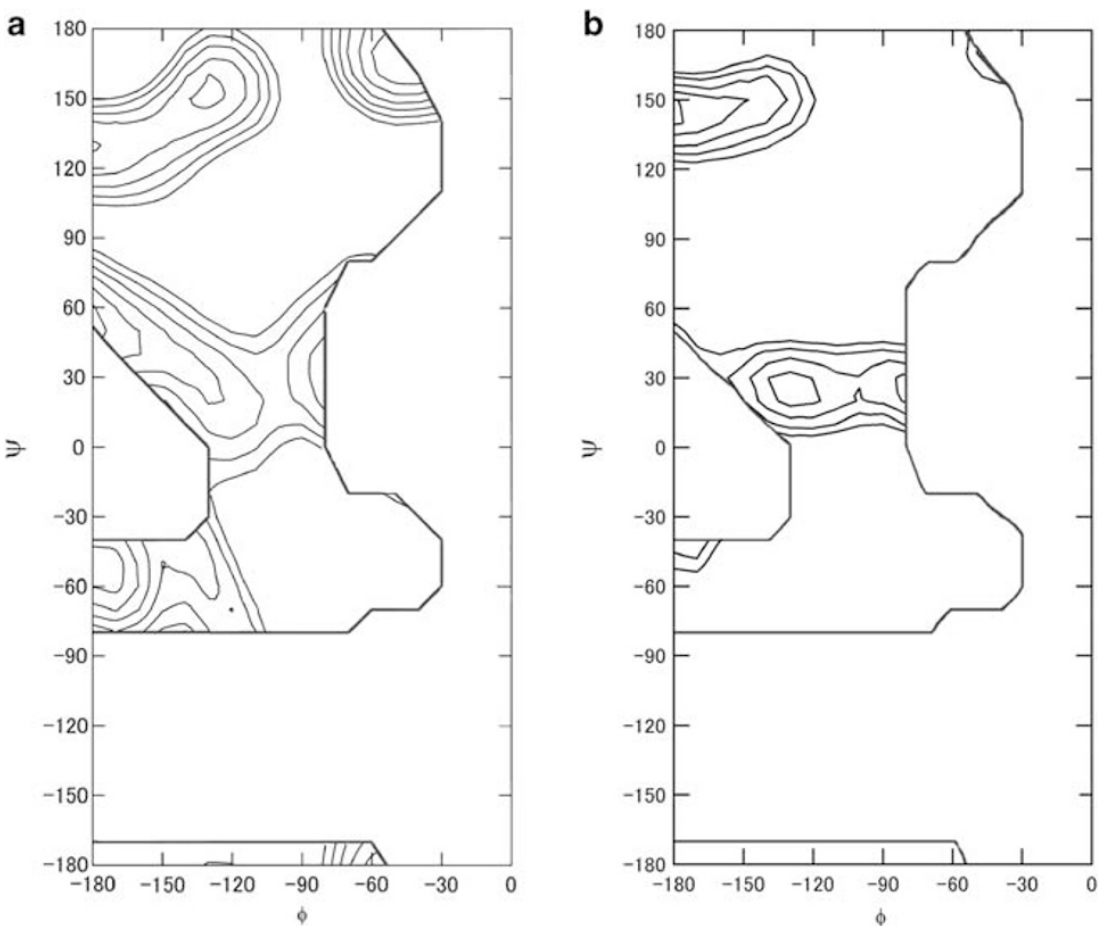

Figure 5 RMSD contour plot for the 2D spin-diffusion spectra for (a) P1 and (b) P3 in the energetically allowed region. The torsion angles $(\varphi, \psi)=\left(-130^{\circ}\right.$, $\left.150^{\circ}\right)$ for $\mathrm{P} 1$ and $\left(-180^{\circ}, 140^{\circ}\right)$ for $\mathrm{P} 3$ gave agreement between the observed and calculated $2 \mathrm{D}$ spin-diffusion spectra (Figure 4$)$ in the $\beta$-sheet-favored region, although the agreement is relatively poor for $\mathrm{P} 1$. 


\section{ACKNOWLEDGEMENTS}

TA acknowledges support from Grant-in-Aid for Scientific Research from Ministry of Education, Science, Culture and Supports of Japan (23245045) and (21550112). We would like to thank the Nissan Chemical Industry Ltd for kind help with the PDSD NMR measurement.

1 Vollrath, F. \& Knight, D. P. Liquid crystalline spinning of spider silk. Nature 410 , 541-548 (2001).

2 Shao, Z. \& Vollrath, F. Materials: Surprising strength of silkworm silk. Nature $\mathbf{4 1 8}$ 741-741 (2002).

3 Xu, M. \& Lewis, R. V. Structure of a protein superfiber: spider dragline silk. Proc. Natl. Acad. Sci. USA 87, 7120 (1990).

4 Hinman, M. B. \& Lewis, R. V. Isolation of a clone encoding a second dragline silk fibroin. Nephila clavipes dragline silk is a two-protein fiber. J. Biol. Chem. 267, 19320 (1992).

5 Peng, X., Shao, Z., Chen, X., Knight, D. P., Wu, P. \& Vollrath, F. Further investigation on potassium-induced conformation transition of Nephila spidroin film with two-dimensional infrared correlation spectroscopy. Biomacromolecules 6, 302-308 (2005).

6 Chen, X., Knight, D. P. \& Vollrath, F. Rheological characterization of Nephila spidroin solution. Biomacromolecules 3, 644-648 (2002).

7 Dicko, C., Vollrath, F. \& Kenney, J. M. Spider silk protein refolding is controlled by changing pH. Biomacromolecules 5, 704-710 (2004).

8 Yazawa, K., Yamaguchi, E., Knight, D. \& Asakura, T. ${ }^{13} \mathrm{C}$ solid state NMR study of the ${ }^{13} \mathrm{C}$-labeled peptide, (E)8GGLGGQGAG(A)6GGAGQGGYGG as a model for the local structure of Nephila clavipes dragline silk (Masp1) before and after spinning. Biopolymers 97, 347-354 (2012).

9 Rising, A., Johansson, J., Larson, G., Bongcam-Rudloff, E., Engström, W. \& Hjälm, G. Major ampullate spidroins from Euprosthenops australis: multiplicity at protein, mRNA and gene levels. Insect Mol. Biol. 16, 551-561 (2007).

10 Asakura, T., Ashida, J., Yamane, T., Kameda, T., Nakazawa, Y., Ohgo, K. \& Komatsu, K. A repeated $\beta$-turn structure in poly (Ala-Gly) as a model for silk I of Bombyx mori silk fibroin studied with two-dimensional spin-diffusion NMR under off magic angle spinning and rotational echo double resonance1. J. Mol. Biol. 306, 291-305 (2001).

11 Asakura, T., Yao, J., Yamane, T., Umemura, K. \& Ulrich, A. S. Heterogeneous structure of silk fibers from Bombyx mori resolved by ${ }^{13} \mathrm{C}$ solid-state NMR spectroscopy. J. Am. Chem. Soc. 124, 8794-8795 (2002).

12 Ohgo, K., Niemczura, W. P., Ashida, J., Okonogi, M., Asakura, T. \& Kumashiro, K. K. Heterogeneity in the conformation of Valine in the elastin mimetic (LGGVG) 6 as shown by solid-state ${ }^{13} \mathrm{C}$ NMR spectroscopy. Biomacromolecules 7, 3306-3310 (2006).

13 Ashida, J., Ohgo, K. \& Asakura, T. Determination of the torsion angles of alanine and glycine residues of Bombyx mori silk fibroin and the model peptides in the silk I and silk II forms using 2D spin diffusion solid-state NMR under off magic angle spinning. J. Phys. Chem. B 106, 9434-9439 (2002).

14 Asakura, T., Ashida, J. \& Ohgo, K. Conformational characterization of (Val-Pro-Gly-ValGly) ${ }_{6}$ with ${ }^{13} \mathrm{C}$ solid state NMR. Polym. J. 35, 293-296 (2003).

15 Ashida, J., Ohgo, K., Komatsu, K., Kubota, A. \& Asakura, T. Determination of the torsion angles of alanine and glycine residues of model compounds of spider silk $(A G G)_{10}$ using solid-state NMR methods. J. Biomol. NMR 25, 91-103 (2003).

16 Asakura, T., Yang, M., Kawase, T. \& Nakazawa, Y. ${ }^{13} \mathrm{C}$ solid-state NMR study of structural heterogeneity in peptides containing both polyalanine and repeated GGA sequences as a local structural model of Nephila clavipes dragline silk (spidroin 1). Macromolecules 38, 3356-3363 (2005).

17 Ohgo, K., Kawase, T., Ashida, J. \& Asakura., T. Solid-state NMR analysis of a peptide (Gly-Pro-Gly-Gly-Ala)6-Gly derived from a flagelliform silk sequence of Nephila clavipes. Biomacromolecules 7, 1210-1214 (2006).

18 Nakazawa, Y. \& Asakura, T. Structure determination of a peptide model of the repeated helical domain in Samia cynthia ricini silk fibroin before spinning by a combination of advanced solid-state NMR methods. J. Am. Chem. Soc. 125, 7230-7237 (2003).

19 Nakazawa, Y., Bamba, M., Nishio, S. \& Asakura, T. Tightly winding structure of sequential model peptide for repeated helical region in Samia cynthia ricini silk fibroin studied with solid-state NMR. Protein Sci. 12, 666-671 (2003).

20 Ramachandran, G. N., Venkatachalam, C. M. \& Krimm, S. Stereochemical criteria for polypeptide and protein chain conformations: III helical and hydrogen-bonded polypeptide chains. Biophysical journal 6, 849-872 (1966). 